\title{
Portal vein thrombosis - a primer for the general physician
}

\author{
Authors: Mohammad Harris ${ }^{A}$ and Jecko Thachil ${ }^{B}$
}

Portal vein thrombosis (PVT) is thrombosis of the portal circulation, which may present with varied symptoms. However, increased use of abdominal imaging has noted a large number of incidental PVT. At the same time, PVT may be overlooked in the absence of the characteristic symptoms. More recently, a JAK2 mutation has been recognised as a cause of PVT. Anticoagulation is the mainstay of treatment, but can pose a dilemma because of the associated coagulopathy, the presence of varices and thrombocytopenia.

KEYWORDS: Portal vein thrombosis

\section{Introduction}

Portal vein thrombosis (PVT) develops in the trunk of the portal vein itself or in either the right or left intrahepatic branches, sometimes extending down to the superior mesenteric or splenic veins. The first case of PVT was described in 1868 by Balfour and Stewart in a patient with ascites, splenomegaly and variceal dilation. ${ }^{1}$ An overview of the anatomy of the portal vein is useful to understand the aetiological factors for this complication and its clinical consequences (Fig 1). The portal vein originates posterior to the neck of the pancreas from the confluence of the superior mesenteric vein and the splenic vein. It supplies approximately $75 \%$ of the blood to the liver and is a valve-less vessel. Its predominant function is to carry (porta $=$ to carry) nutrientrich blood from the gastrointestinal tract to the liver.

\section{Is the incidence of PVT on the rise?}

PVT is a rare clinical diagnosis in the current era. A study of almost 24,000 autopsies in Sweden revealed a prevalence of $1 \%{ }^{2}$ However, increased use of imaging for other purposes have noted an increased number of diagnoses of what may be termed 'incidental PVT'. For example, a large case series of 701 patients with cirrhosis undergoing investigation using routine Doppler ultrasound found a prevalence rate of $11.2 \%$ (79 patients). ${ }^{3}$ Another prospective study following 73 patients with cirrhosis found PVT diagnosis in 12 patients (16.4\%) within 1 year. $^{4}$ In our own institution, out of a total of 82 patients diagnosed with a PVT, 36 were incidental diagnoses while 43 patients had

Authors: ${ }^{\text {A }}$ medical student, University of Manchester, Manchester, UK; ${ }^{B}$ consultant haematologist, Manchester Royal Infirmary, Manchester, UK long-standing PVT and only three patients had acute PVT. The difference in prevalence rates according to the modality used for diagnosis can be seen when comparing studies. A rate of $0.6 \%$ has been reported when using angiography ${ }^{5}$ compared with $4.4 \%$ with ultrasound ${ }^{6}$ and $11 \%$ with computerised tomography (CT) and magnetic resonance imaging (MRI). ${ }^{3}$ In the 2016 Italian study (PRO-LIVER), the prevalence of ultrasounddetected PVT was $17 \%$ in patients with cirrhosis, but $43 \%$ of the cases were asymptomatic. ${ }^{7}$ In summary, the incidence of PVT diagnosis is increasing because of the higher number of abdominal imaging studies performed in hospitals.

\section{Do we classify PVT as acute and chronic?}

Different methods of PVT categorisation have been reported in the literature with some based on chronicity and others according to the occlusive capacity of the thrombus. What is definitely lacking is a clear definition for what constitutes an 'acute PVT'. It is generally believed that PVT may be considered acute if the symptoms appear less than 60 days prior to hospital assessment. ${ }^{8}$ A formal classification system used for PVT is the Yerdel classification, which considers both the degree and extension of the thrombosis (Box 1). ${ }^{9}$ PVT can also be categorised according to the presence or absence of cavernoma

\section{Box 1. Classifications of portal vein thrombosis}

\section{(PVT)}

Based on extent of thrombosis

1 Minimally thrombosed - the thrombus covers less than $50 \%$ of the diameter of the lumen.

2 Total occlusion - greater than $50 \%$ of the lumen is occluded but with minimal extension into the superior mesenteric vein.

3 Complete thrombosis of the portal vein and the proximal superior mesenteric vein.

4 Complete thrombosis of the portal vein and the proximal and distal superior mesenteric vein.

Based on associated liver disease

1 Type I - partial PVT without cavernoma

2 Type II - partial PVT associated with cavernoma

3 Type III - complete PVT without cavernoma

4 Type IV - complete PVT associated with cavernoma

Adapted from Yerdel et al ${ }^{9}$ and Ma et al. ${ }^{10}$ 


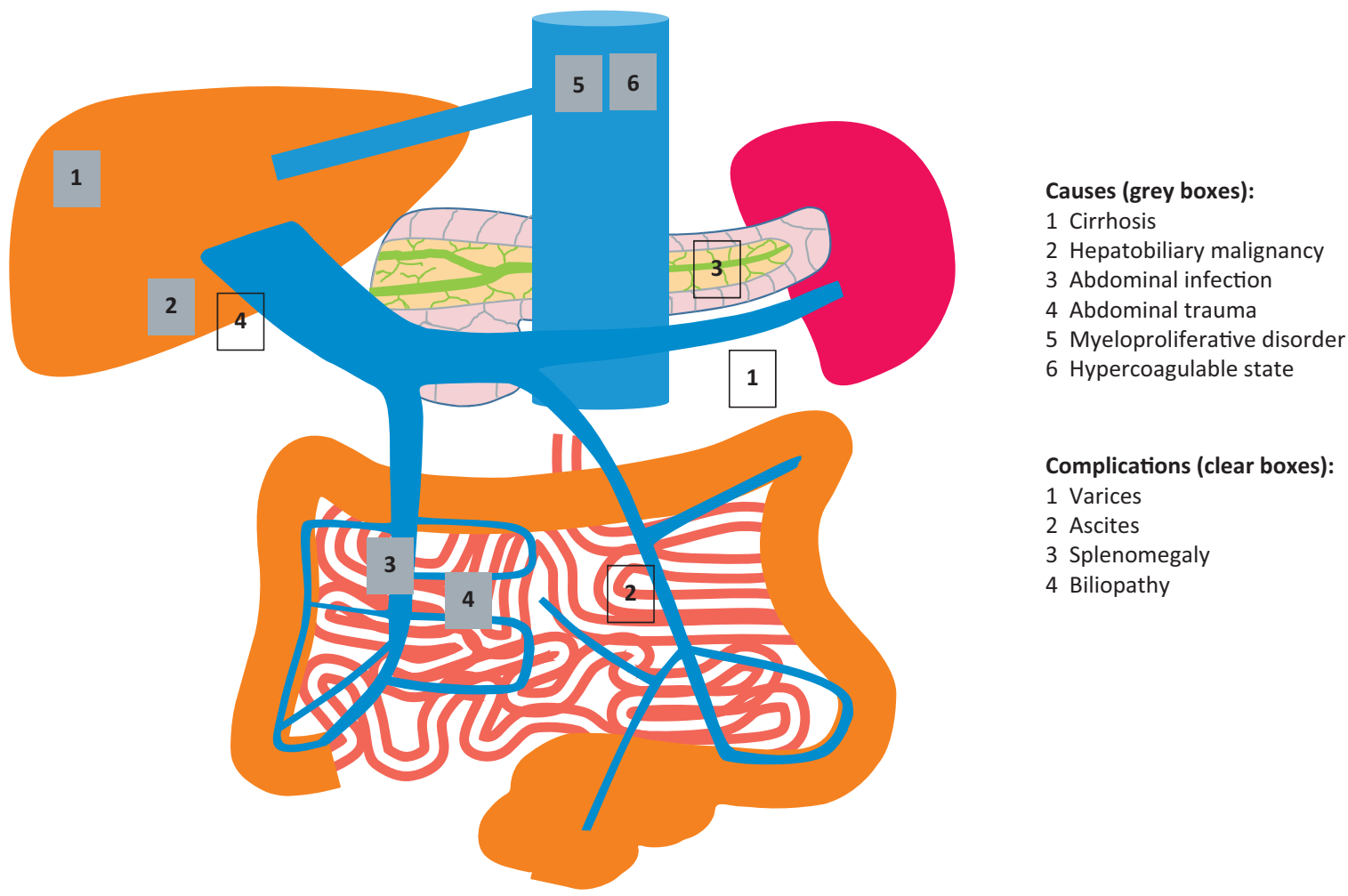

Fig 1. Causes and complications of portal vein thrombosis.

in combination with the degree of occlusion of the portal blood supply. ${ }^{10}$

\section{Aetiopathogenesis of PVT}

PVT can develop similar to the formation of a thrombus in any other vessel. This can be explained by the Virchow's triad - endothelial damage, stasis and hypercoagulability. ${ }^{11}$ Endothelial damage can occur in patients who have a liver pathology (eg cirrhosis) or because of vessel damage from a surgical procedure. Stasis can be secondary to a tumour compressing the portal vein and also sluggish flow in this vessel because of other reasons. Since the portal vein is the principal transporter of nutrients and other components from the intestinal circulation to the liver for metabolism, any factors that may slow down this flow could lead to PVT (eg intestinal infections). Lastly, some haematological conditions, including hereditary thrombophilia, can increase the risk of PVT by being a hypercoagulable state.

\section{Risk factors for PVT}

PVT has been shown to be associated with a variety of different conditions, including cirrhosis, myeloproliferative disorders and abdominal infections (Fig 1). The underlying cause can be found in up to $80 \%$ of cases with a comprehensive search. ${ }^{12,13}$ A Swedish study of almost 24,000 autopsies found patients with PVT also have cirrhosis (28\%), hepatobiliary malignancy (primary 23\%, secondary 44\%), abdominal infections (10\%) and myeloproliferative disorders (MPD) (3\%). ${ }^{1}$ However, 14\% of patients had no associated condition that could be linked to the development of PVT. In general, the risk factors for PVT can be divided into local and systemic.

\section{Local}

In non-cirrhotic PVT, the risk factors can be broadly split into two categories: local and general. Of those where a risk factor is present, local factors account for up to $30 \%$ of cases and general factors for up to $70 \% .{ }^{14}$ Local factors include direct injury to the portal vein. This may be during a splenectomy or hepatectomy, insertion of a surgical shunt or any other surgical procedure in the vicinity of the portal vein. ${ }^{12,15}$ Inflammation of any of the abdominal organs is another local factor; ${ }^{16}$ this includes pancreatitis, cholecystitis, appendicitis and any other intra-abdominal infection. One study looking at 102 patients with non-cirrhotic non-malignant acute PVT showed local factors to be the cause in up to $21 \%$; the most common of them being acute pancreatitis. ${ }^{17}$ In children of the Asia-Pacific region, three factors have been seen to have a significant effect. These are omphalitis, neonatal umbilical sepsis and umbilical vein cannulation. ${ }^{18}$ PVT due to umbilical vein cannulation is common but resolves spontaneously in most cases. ${ }^{19}$

\section{Systemic}

Of the many systemic risk factors, MPD are the most common occurring in up to $40 \%$ of cases. ${ }^{17}$ Other genetic mutations causing hypercoagulability also increase the risk. These include factor V Leiden mutation and the G20210A mutation of the 
prothrombin gene, which is reported to be present in up to $32 \%$ of patients with PVT. ${ }^{20}$ Other hereditary thrombophilic mutations - such as the deficiency of natural anticoagulants like protein $\mathrm{C}, \mathrm{S}$ and antithrombin - have been suggested as 'causative' factors for PVT. However, this may be controversial because of the fact that these endogenous anticoagulants are synthesised in the liver and their levels are likely to decrease along with synthetic liver function. If there is family history of thrombosis and these enzyme levels have been found to be deficient in the family members, they may be considered as pathological. Mutations in the methylene tetrahydrofolate reductase (MTHFR) gene and Plasminogen activator inhibitor-type 1 (PAI-1) gene have also been considered to predispose to PVT. In some patients, multiple mutations may be present. ${ }^{21}$ Paroxysmal nocturnal haemoglobinuria is a rare haematological disorder that can also increase the risk of PVT. In all patients who develop PVT in the absence of a clear local risk factor, lactate dehydrogenase - a marker of haemolysis should be done and if abnormal, haematology consultation should be sought for detailed tests for this condition. Recent pregnancy can also increase the risk, as can antiphospholipid syndrome, recent oral contraceptive use and cytomegalovirus infection. ${ }^{12,22}$

\section{JAK2 mutation and PVT}

The Janus kinase 2 (JAK2) V617F gene mutation is a recently described abnormality that is characteristically seen in many patients with MPD. A meta-analysis of MPD in patients with PVT reported a mean prevalence of $31.5 \% .{ }^{23}$ One study showed the figure to be $30.7 \%$, with $16.7 \%$ being occult MPD and $13.8 \%$ being classical MPD. ${ }^{24}$ Another recent study, looking at 44 patients, reported PVT to be the first manifestation of MPD in 31 patients (70\%). ${ }^{25}$ However, this mutation is not very common in those with PVT secondary to liver disease. It was shown to be present in around $25 \%$ with non-cirrhotic non-malignant PVT compared with $1.4 \%$ in those with cirrhosis. ${ }^{26,27}$ It is important to note that the identification of the JAK2 mutation has increased the diagnosis of MPD as the cause of PVT significantly. Some have reported it to have had an effect as great as $20 \% .^{28,29}$ The authors' practice is to check for this mutation in all patients who do not have a clear local cause for the PVT. If JAK2 mutation is detected, then a haematology consult is sought even in the absence of MPD because positive JAK2 mutation may have implications for management.

\section{Do we miss cases of PVT?}

Presentation of PVT can be non-specific (Box 2). Colicky pain that is not localised can be the presenting feature of PVT and the manifestation of intestinal ischaemia. ${ }^{11}$ Similarly, nausea and vomiting along with loss of appetite can be the features of PVT with complications. ${ }^{14}$ Occult blood in stool has also been the initial clue for the diagnosis of PVT rarely. Unexplained splenomegaly is another presentation that necessitates an ultrasound Doppler to rule out PVT. ${ }^{11}$ In some of these cases of splenomegaly, there will be cytopenias due to pooling of the blood in the spleen. In the absence of complications, PVT is often picked up incidentally on a radiological test that was not intended to diagnose the condition. In any patient who has been diagnosed with PVT, continuous monitoring to look for

\section{Box 2. Clinical presentations of portal vein}

\section{thrombosis}

> Right hypochondrial pain - acute onset or long-standing

Splenomegaly with ascites

$>$ Unexplained abdominal pain

$>$ Features of intestinal ischaemia

$>$ Occult blood in stool

$>$ Unexplained gatro-oesophageal varices

Abrupt worsening of ascites or hepatic encephalopathy

signs of intestinal ischaemia is important. These may be newonset fever, continuing abdominal pain or rising inflammatory markers despite anticoagulation. ${ }^{11,14,24}$

\section{Diagnosis of PVT}

\section{Imaging}

The most common imaging technique used for the diagnosis of PVT is Doppler ultrasound, with a very high sensitivity in most studies (Fig 2). ${ }^{30}$ However, there are some drawbacks to the use of ultrasound techniques. If the patient is obese or there is significant bowel gas, the visualisation may be poor. It is also a poor method of identifying thrombi in the splenic and superior mesenteric veins. Finally, assessing bowel ischaemia (which may occur secondary to mesenteric vein thrombosis) is very difficult. ${ }^{30} \mathrm{CT}$ and MRI have the additional advantages of being able to define the extent of the thrombus, show any evidence of bowel ischaemia/infarction and for looking at other organs surrounding the portal vein. ${ }^{30,31}$ The sensitivity and specificity of these techniques have been shown to be $100 \%$ and $98 \%$, respectively, in patients awaiting a liver transplant. ${ }^{32} \mathrm{New}$ techniques like magnetic resonance portography are especially useful in patients who are severely debilitated, in those who have difficult venous access and for patients with renal failure. ${ }^{33}$

\section{Additional imaging}

In patients with strong suspicion for PVT and when Doppler ultrasound is negative, it is important to perform additional imaging to rule out the diagnosis. This may be a CT abdomen or one of the newer techniques. In addition, in patients who are confirmed to have PVT, further imaging may be necessary to look for: ${ }^{30}$

$>$ any signs of cirrhosis as the cause of any PVT

$>$ extent of the thrombus, especially extension into the mesenteric or splenic veins

$>$ splenomegaly

$>$ liver pathologies

$>$ structures around the portal vein

$>$ signs of cavernous transformation.

In patients with fully thrombosed PVT, the fibroblasts form a firm, collagenous plug that develops tortuous veins. The plug is termed a 'portal cavernoma'. This transformation usually begins within days of acute thrombus formation and develops over many months. ${ }^{14}$ As a PVT progresses and becomes a portal cavernoma, hepatocellular collaterals connect the 


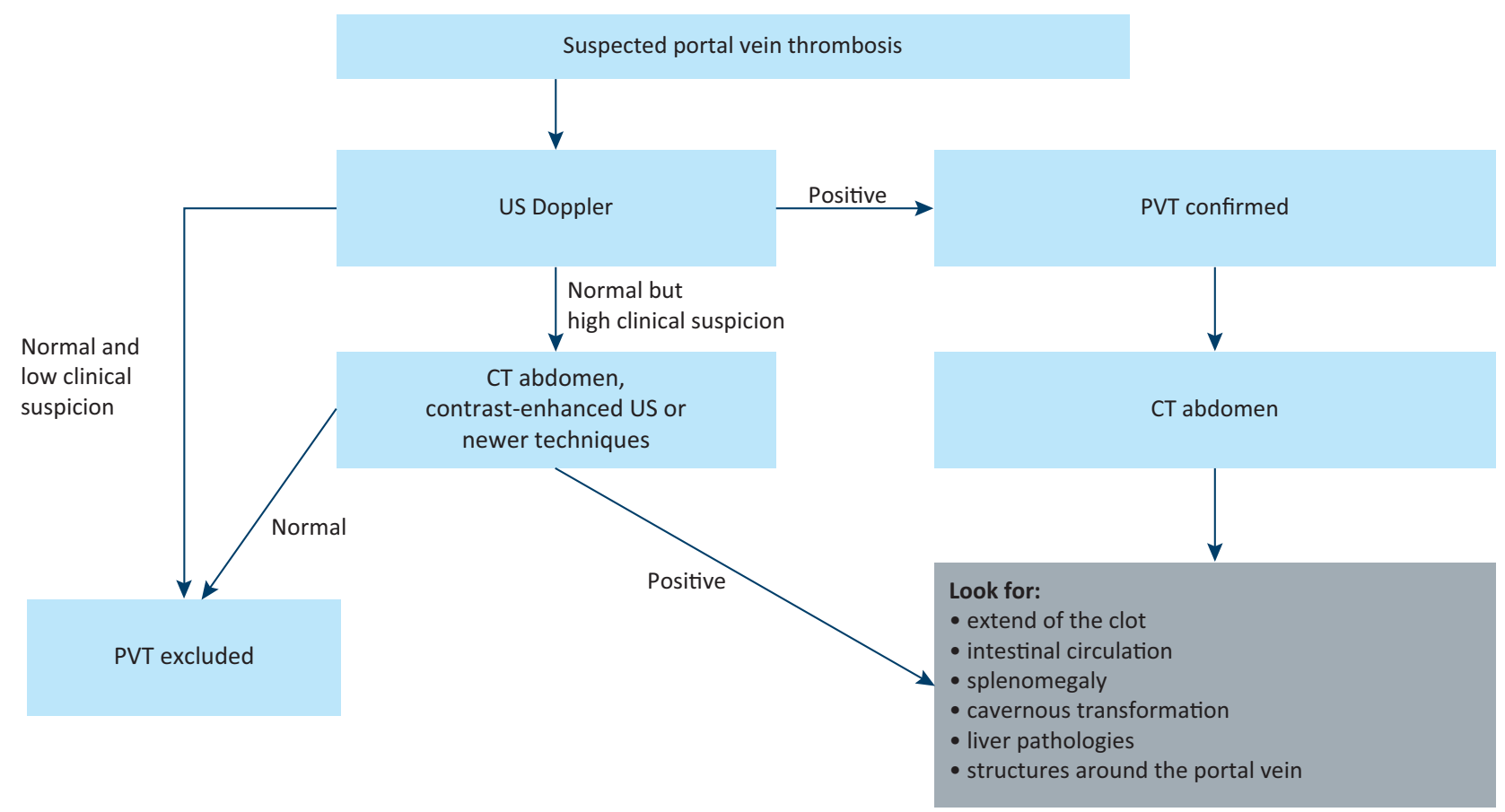

Fig 2. Imaging strategy for portal vein thrombosis. CT = computerised tomography; PVT = portal vein thrombosis; US = ultrasound

patent portions of the vein before and after the thrombus. The number of these collaterals varies from person to person. Portal cholangiopathy or portal biliopathy may also form with compression or deformation of the large bile ducts. The pancreatic veins may enlarge if there is occlusion at the origin of the portal vein. ${ }^{34}$

\section{Endoscopy}

All patients with PVT should undergo a gastrointestinal endoscopy to exclude the presence of varices, which may be in the oesophagus or the gastric mucosa. ${ }^{10,14}$ In patients with PVT caused by concomitant liver disease, portal hypertensive gastropathy is often observed while in those with long-standing PVT, gastric varices are seen in nearly half of the patients. ${ }^{35}$ These varices need to be identified because they are important in planning management for the following reasons:

$>$ need for anticoagulation has to be balanced with the bleeding risk from the varices

$>$ choice of anticoagulation may depend on the bleeding risk of varices and reversibility of the anticoagulant

$>$ ability to 'cure' the varices may impact on long-term anticoagulation management

$>$ some researchers recommend continued anticoagulation to prevent variceal progression.

\section{Blood tests}

Blood tests do not usually show anything significant to suggest PVT (Box 3). Liver function tests are usually normal unless there is cirrhosis or any other liver disease. ${ }^{10}$ The alkaline phosphatase may rise, however, if there is portal hypertensive biliopathy developing as a complication. As is generally the case in venous thrombosis, D-dimer is usually increased and not helpful. ${ }^{24}$ It is important to exclude an underlying systemic disorder in those with no local cause identified. All patients without a clearly identifiable cause on imaging should have a JAK2 mutation analysis, lactate dehydrogenase and tests for paroxysmal nocturnal haemoglobinuria, factor V Leiden and prothrombin gene mutations. If anticoagulant enzyme deficiency is suspected based on family history, these tests are not performed in the acute setting but after an interval of at least 3-6 months and should not be performed while the patient is receiving injectable anticoagulants (low molecular weight heparin) as oral anticoagulants can affect the results. Tests for antiphospolipid syndrome and vasculitides are also appropriate.

\section{Box 3. Prothrombotic conditions that need to be} investigated in patients with portal vein thrombosis
> JAK2 mutation
> Factor $\mathrm{V}$ Leiden mutation
Prothrombin gene mutation
$>$ Antithrombin deficiency
$>$ Protein $\mathrm{C}$ and $\mathrm{S}$ deficiency
$>$ Antiphospholipid syndrome
> Paroxysmal nocturnal haemoglobinuria
$>$ Vasculitis, including Behçet's disease
> Methylene tetrahydrofolate reductase and plasminogen activator inhibitor-type 1 gene mutations 
Box 4. Management of acute portal vein thrombosis by the on-call physician

1 Assess for bleeding risk - varices, concomitant antiplatelet agents etc

2 If bleeding risk is low, start therapeutic anticoagulation with low molecular weight heparin

3 If varices likely to be present (history of haematemesis or known cirrhosis), refer to gastroenterology to see if definitive treatment is possible (eg banding) and commence anticoagulation when safe from bleeding risk point of view

4 If symptoms suggest or imaging confirms intestinal ischaemia, refer to surgeons. Start intravenous unfractionated heparin since interruption of the anticoagulation may be required for surgery. Monitor the heparin infusion with anti-Xa if APTT is abnormal prior to commencement because of concomitant liver disease

5 If the thrombus extends while on adequate therapeutic anticoagulation, consideration should be given for thrombolysis. If very high bleeding risk is a contraindication for thrombolysis, surgical intervention may be sought

6 In patients with decompensated cirrhosis and portal hypertension, consideration should be made for a transjugular intrahepatic portosystemic shunt (TIPS) procedure

\section{Management of PVT}

The aims of management of PVT are twofold (Box 4) - to prevent the progression of the thrombus and to recanalise the thrombosed veins, which will prevent the complications of portal hypertension and mesenteric ischaemia (Fig 3). Three main methods have been attempted

1 anticoagulation

2 thrombolysis

3 interventions like the transjugular intrahepatic portosystemic shunt (TIPS) procedure.

We generally follow the American Association for the Study of Liver Disease (AASLD) guidelines on the management of PVT. $^{11}$

\section{Anticoagulation}

It would seem logical to treat PVT to prevent thrombus progression and new clot formation. A 2011 systematic review showed that more than $80 \%$ of acute PVT cases do not resolve without the use of anticoagulation. ${ }^{36}$ And for those that do recanalise without such drugs, it is either due to the spontaneous resolution of a self-limiting illness, such as acute pancreatitis, or due to the fact that the thrombosis load was very small. ${ }^{36}$ In the systematic review, the time taken for recanalisation ranged from a few days to over 6 months indicating, in some cases, long-term anticoagulation may be needed for recanalisation. ${ }^{36}$ With respect to complications, despite the presence of varices in 47 patients, five suffered a variceal bleed and all five of these cases failed to recanalise giving another reason to consider anticoagulation despite the presence of varices. ${ }^{36}$ Anticoagulation has also been shown to reverse biliary complications caused by a PVT. ${ }^{37}$

The purpose of anticoagulation is not only to allow recanalisation of the thrombosed vessel but to prevent thrombus extension. ${ }^{38}$ A 2010 multicentre study, which followed up patients for 2 years, noted that anticoagulation

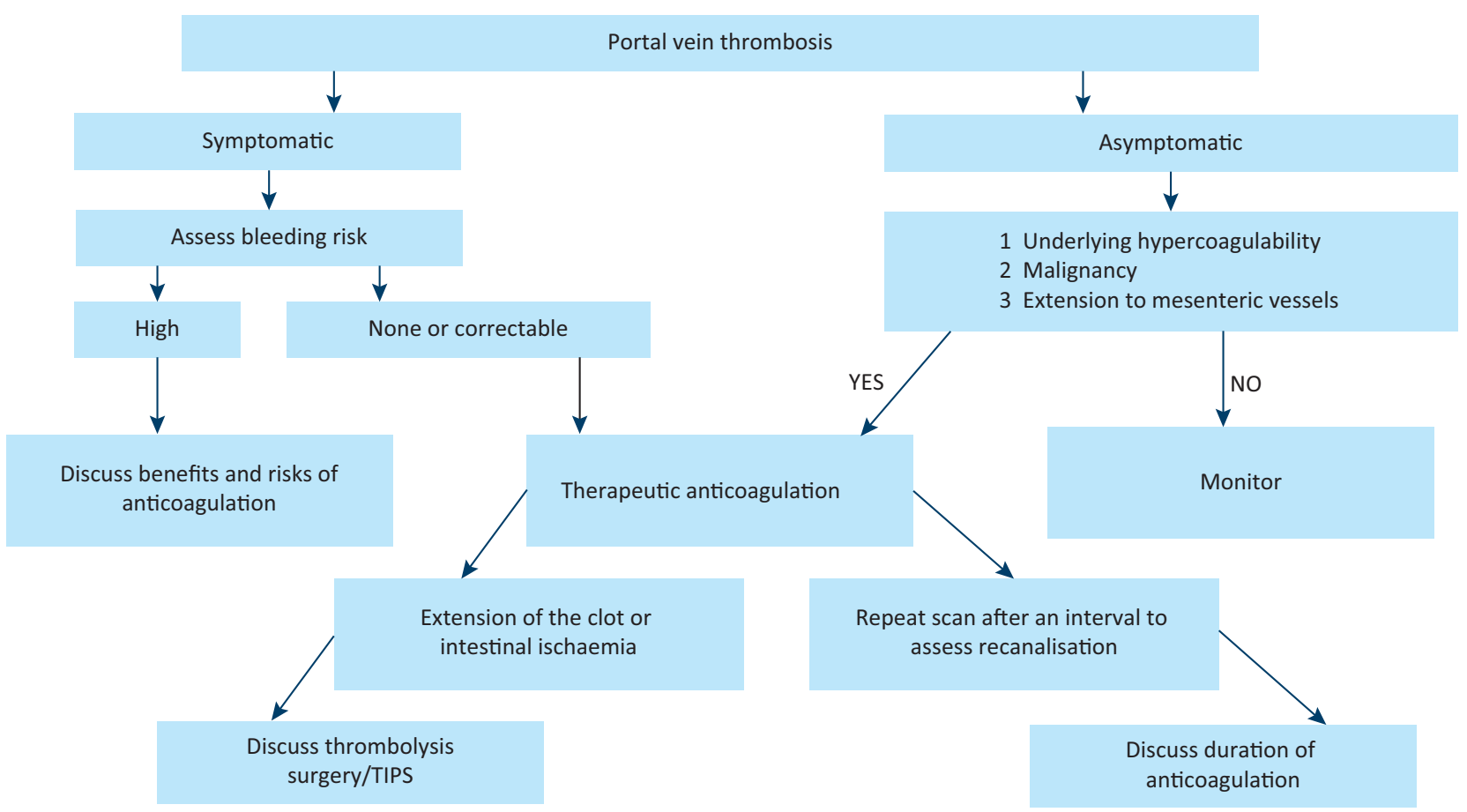

Fig 3. Management options for portal vein thrombosis. 
did not have any effect on recanalisation beyond 6 months. They found two independent factors predicted the failure of recanalisation: presence of ascites and hypercoagulability. ${ }^{39}$ Anticoagulating early seems to have the most effect in this respect. Delaying until the second week decreases the rate of recanalisation from $69 \%$ to $25 \% .{ }^{38}$ Early anticoagulation also minimises the risk of serious complications, such as bowel necrosis leading to peritonitis, developing. ${ }^{40}$

Although 6 months of anticoagulation may be adequate in many patients, it may be beneficial to continue anticoagulation for longer than 6 months in some cases. These cases include patients with an underlying systemic prothrombotic disorder, those with a personal and family history of venous thromboses, those with a history of recurrent thrombosis and also those with a JAK2 mutation. ${ }^{41}$ Anticoagulation may increase the risk of bleeding but it does not have any effect on the severity of the bleed. ${ }^{42,43}$

\section{Thrombolysis}

In certain cases of PVT, despite anticoagulation, the patient continues to deteriorate - especially with extension of the thrombus into the mesenteric vessels. Such patients should be considered for thrombolysis. ${ }^{11}$ In addition, those PVT patients who present with ischaemia should also be thrombolysed. ${ }^{11}$ Thrombolysis can be performed via the superior mesenteric artery using intra-arterial infusion or directly into the portal vein using a catheter inserted via the transhepatic or transjugular route. ${ }^{44}$ If a patient has developed bowel necrosis, surgical exploration is necessary.

\section{Transjugular intrahepatic portosystemic shunt}

TIPS is a technique where a portal flow shunt is used to recanalise the portal vein and prevent the recurrence of the thrombosis. It is a very challenging procedure and is not recommended other than in experienced centres. ${ }^{11,45-47}$ It also cannot be used if the occluded portal vein cannot be catheterised. The complications of TIPS include hepatic encephalopathy, TIPS dysfunction and the possibility of misplacement. This procedure may be considered if anticoagulation is contraindicated or in specialised institutions when the portal hypertension symptoms worsen despite anticoagulation.

\section{Dilemmas in the management of PVT}

\section{Choice of anticoagulant}

Although anticoagulation is the cornerstone of PVT management, there may be issues in the choice of the anticoagulant agent. Because patients with liver disease may have abnormal coagulation tests, monitoring an oral anticoagulant like warfarin may not be possible. However, low molecular weight heparin can be used at weight-based doses without any monitoring. In the case of coagulation tests becoming normal (and this was due to PVT), the injectable drugs can be switched to warfarin. There is currently no data to recommend the use of newer oral anticoagulants in this setting; they are contraindicated in patients with severe liver impairment.

\section{Presence of coagulopathy and thrombocytopenia}

Traditionally, cirrhosis was considered to cause a hypocoagulable state with autoanticoagulation being characteristically attributed to severe liver impairment. However, a relatively simultaneous reduction in both proand anticoagulant proteins causes a degree of balance of haemostasis in most patients. ${ }^{48}$ The coagulation screen only measures the procoagulant aspect and not the anticoagulant proteins. Hence, abnormal prothrombin time and activated partial thrombopastin time (APTT) by themselves should not be considered a contraindication for anticoagulation in PVT. ${ }^{49}$ Thrombocytopenia in cirrhosis causes a paradox. The low platelet would be expected to cause bleeding yet it has been shown to be a risk factor for the development of a PVT. ${ }^{50}$ In relation to commencing anticoagulation, a platelet count more than $50 \times 10^{9} / \mathrm{L}$ is considered safe. ${ }^{51}$ Lower thresholds may be used but require careful inpatient monitoring for any bleeding. ${ }^{50}$ It is possible that the pooling of platelets in the spleen contributes to thrombocytopenia and this should not preclude anticoagulation.

\section{The patient with varices}

In all patients with confirmed PVT, performing a gastrointestinal endoscopy may be considered good practice. If varices are identified, measures should be adopted to treat them (if possible).$^{51}$ However, there may be occasions where the variceal treatment may not be possible, anticoagulation may need to be started before the endoscopy or the patient may be at risk of bowel ischaemia that requires surgery. In such cases, a bleeding risk assessment should be made and the patient treated with intravenous heparin. Monitoring the coagulation test with APTT may be tricky in these patients (they may have been abnormal before heparin is administered), in which case monitoring using anti-Xa assay should be considered with discussions with the haematologist. Intravenous heparin has the benefit of easy reversibility, which is certainly attractive in the patient scenarios discussed. Once the patient is stabilised, the intravenous heparin can be converted to low molecular weight heparin.

\section{The asymptomatic patient}

Although anticoagulation is definitely the treatment of choice, concerns may arise in those cases where PVT has appeared as an incidental finding during imaging. Unfortunately, there are no concrete answers as of yet because of the lack of randomised control trials of the different management options in this scenario. One option is to manage the patient conservatively. This will obviously depend on the status of the patient and the stage of the PVT. In the absence of an underlying malignancy or prothrombotic condition or extension outside the portal vein, it may be reasonable to adopt a 'watch and wait' option. ${ }^{52}$ Anticoagulation may be commenced at the earliest signs of any deterioration.

\section{Conclusion}

PVT may present with very minimal symptoms and is increasingly being identified on imaging studies performed 
for other indications. The clinical symptoms are not often classical but active search and surveillance for mesenteric extension of thrombosis is important. A search for causes is critical in patients with no associated liver disease, especially for hypercoagulability and JAK2 mutations. Imaging should also aid in identifying the cause and possible complications. Although anticoagulation is the mainstay of treatment, it may not be necessary in all cases and could pose a dilemma in certain scenarios.

\section{Conflicts of interest}

The authors have no conflicts of interest to declare.

\section{References}

1 Balfour G, Stewart T. Cases of enlarged spleen complicated with ascites, both depending upon varicose dilatation and thrombosis of the portal vein. Edinb Med J 1869;14:589-98.

2 Ögren M, Bergqvist D, Björck M et al. Portal vein thrombosis: prevalence, patient characteristics and lifetime risk: a population study based on 23,796 consecutive autopsies. World J Gastroenterol 2006;12:2115-9.

3 Amitrano L, Guardascione MA, Brancaccio V et al. Risk factors and clinical presentation of portal vein thrombosis in patients with liver cirrhosis. J Hepatol 2004;40:736-41.

4 Zocco MA, Di Stasio E, De Cristofaro R et al. Thrombotic risk factors in patients with liver cirrhosis: correlation with MELD scoring system and portal vein thrombosis development. J Hepatol 2009;51:682-9.

5 Okuda K, Ohnishi K, Kimura K et al. Incidence of portal vein thrombosis in liver cirrhosis. An angiographic study in 708 patients. Gastroenterology 1985;89:279-86.

6 Gaiani S, Bolondi L, Li Bassi S et al. Prevalence of spontaneous hepatofugal portal flow in liver cirrhosis. Clinical and endoscopic correlation in 228 patients. Gastroenterology 1991;100: 160-7.

7 Violi F, Corazza RG, Caldwell SH et al. Portal vein thrombosis relevance on liver cirrhosis: Italian Venous Thrombotic Events Registry. Intern Emerg Med 2016;11:1059-66.

8 Malkowski P, Pawlak J, Michalowicz B et al. Thrombolytic treatment of portal thrombosis. Hepatogastroenterology 2003;50:2098 100.

9 Yerdel MA, Gunson B, Mirza D et al. Portal vein thrombosis in adults undergoing liver transplantation: risk factors, screening, management, and outcome. Transplantation 2000;69:1873-81.

10 Ma J, Yan Z, Luo J et al. Rational classification of portal vein thrombosis and its clinical significance. PLoS One 2014;9:e112501.

11 DeLeve LD, Valla DC, Garcia-Tsao G; American Association for the Study Liver Diseases. Vascular disorders of the liver. Hepatology 2009;49:1729-64.

12 Denninger M-H, Chait Y, Casadevall $\mathrm{N}$ et al. Cause of portal or hepatic venous thrombosis in adults: the role of multiple concurrent factors. Hepatology 2000;31:587-91.

13 Primignani M, Martinelli I, Bucciarelli P et al. Risk factors for thrombophilia in extrahepatic portal vein obstruction. Hepatology 2005;41:603-8.

14 Chawla YK, Bodh V. Portal vein thrombosis. J Clin Exp Hepatol 2015;5:22-40.

15 Fujita F, Lyass S, Otsuka K et al. Portal vein thrombosis following splenectomy: Identification of risk factors. Am Surg 2003;69:951-6.

16 Bernades P, Baetz A, Lévy P et al. Splenic and portal venous obstruction in chronic pancreatitis. Dig Dis Sci 1992;37:340-6.

17 Plessier A, Darwish-Murad S, Hernandez-Guerra M et al. Acute portal vein thrombosis unrelated to cirrhosis: a prospective multicentre follow-up study. Hepatology 2010;51:210-8.
18 Kim JH, Lee YS, Kim SH et al. Does umbilical vein catheterization lead to portal venous thrombosis? Prospective US evaluation in 100 neonates. Radiology 2001;219:645-50.

19 Sakha SH, Rafeey M, Tarzamani MK. Portal venous thrombosis after umbilical vein catheterization. Indian J Gastroenterol 2007;26:283-4.

20 Janssen HL, Meinardi JR, Vleggaar FP et al. Factor V Leiden mutation, prothrombin gene mutation, and deficiencies in coagulation inhibitors associated with Budd-Chiari syndrome and portal vein thrombosis: results of a case-control study. Blood 2000;96:2364-8.

21 Simsek E, Yesilyurt A, Pinarli F, Eyerci N, Ulus AT. Combined genetic mutations have remarkable effect on deep venous thrombosis and/or pulmonary embolism occurence. Gene 2014;536:1716.

22 Amitrano L, Guardascione MA, Scaglione M et al. Acute portal and mesenteric thrombosis: unusual presentation of cytomegalovirus infection. Eur J Gastroenterol Hepatol 2006; 18:443-5.

23 Smalberg JH, Arends LR, Valla DC et al. Myeloproliferative neoplasms in Budd-Chiari syndrome and portal vein thrombosis: a meta-analysis. Blood 2012;120:4921-8.

24 Condat B, Valla D. Nonmalignant portal vein thrombosis in adults. Nat Clin Pract Gastroenterol Hepatol 2006;3:505-15.

25 Hoekstra J, Bresser EL, Smalberg JH et al. Long-term follow-up of patients with portal vein thrombosis and myeloproliferative neoplasms. J Thromb Haemost 2011;9:2208-14.

26 Qi X, Zhang C, Han G et al. Prevalence of the JAK2V617F mutation in Chinese patients with Budd-Chiari syndrome and portal vein thrombosis: a prospective study. J Gastroenterol Hepatol 2012;27:1036-43.

27 Qi X, Yang Z, Bai M et al. Meta-analysis: the significance of screening for JAK2V617F mutation in Budd-Chiari syndrome and portal venous system thrombosis. Aliment Pharmacol Ther 2011;33:1087-103.

28 Kiladjian JJ, Cervantes F, Leebeek FW et al. The impact of JAK2 and MPL mutations on diagnosis and prognosis of splanchnic vein thrombosis: a report on 241 cases. Blood 2008;111:4922-9.

29 Patel RK, Lea NC, Heneghan MA et al. Prevalence of the activating JAK2 tyrosine kinase mutation V617F in the Budd-Chiari syndrome. Gastroenterology 2006;130:2031-8.

30 Berzigotti A, Garcia-Criado A, Darnell A, Garcia-Pagan JC. Imaging in clinical decision-making for portal vein thrombosis. Nat Rev Gastroenterol Hepatol 2014;11:308-16.

31 Parvey HR, Raval B, Sandler CM. Portal vein thrombosis: imaging findings. Am J Roentgenol 1994;162:77-81.

32 Shah TU, Semelka RC, Voultsinos V et al. Accuracy of magnetic resonance imaging for preoperative detection of portal vein thrombosis in liver transplant candidates. Liver Transpl 2006;12:1682-8.

33 Smith CS, Sheehy N, McEniff N, Keogan MT. Magnetic resonance portal venography: use of fast-acquisition true FISP imaging in the detection of portal vein thrombosis. Clin Radiol 2007;62:1180-8.

34 Manzano-Robleda M, Barranco-Fragoso B, Uribe M, MéndezSánchez N. Portal vein thrombosis: What is new? Ann Hepatol 2015;14:20-7.

35 Sarin SK, Lahoti D, Saxena SP, Murthy NS, Makwana UK. Prevalence, classification and natural history of gastric varices: a long-term follow up study in 568 portal hypertension patients. Hepatology 1992;16:1343-9.

36 Hall TC, Garcea G, Metcalfe M, Bilku D, Dennison AR. Management of acute non-cirrhotic and non-malignant portal vein thrombosis: a systematic review. World J Surg 2011;35:2510-20.

37 Louvet A, Texier F, Dharancy S et al. Anticoagulation therapy may reverse biliary abnormalities due to acute portal thrombosis. Dig Dis Sciences 2006;51:11-7.

38 Turnes J, García-Pagan JC, Gonz alez M et al. Portal hypertensionrelated complications after acute portal vein thrombosis: impact of early anticoagulation. Clin Gastroenterol Hepatol 2008;6:1412-7. 
39 Plessier A, Darwish-Murad S, Hernandez-Guerra M et al. Acute portal vein thrombosis unrelated to cirrhosis: a prospective multicenter follow-up study. Hepatology 2010;51:210-8.

40 Turnes J, Garcia-Pagan JC, Gonzalez M et al. Portal hypertensionrelated complications after acute portal vein thrombosis: impact of early anticoagulation. Clin Gastroenterol Hepatol 2008;6:1412-7.

41 Dolovich LR, Ginsberg JS, Douketis JD, Holbrook AM, Cheah G. A meta-analysis comparing low-molecular-weight heparins with unfractionated heparin in the treatment of venous thromboembolism: examining some unanswered questions regarding location of treatment, product type, and dosing frequency. Arch Intern Med 2000;160:181-8.

42 Condat B, Pessione F, Hillaire S et al. Current outcome of portal vein thrombosis in adults: Risk and benefit of anticoagulant therapy. Gastroenterology 2001;120:490-7.

43 Spaander MCW, Hoekstra J, Hansen BE et al. Anticoagulant therapy in patients with non-cirrhotic portal vein thrombosis: effect on new thrombotic events and gastrointestinal bleeding. J Thromb Haemost 2013;11:452-9.

44 Chamarthy MR, Anderson ME, Pillai AK, Kalva SP. Thrombolysis and transjugular intrahepatic portosystemic shunt creation for acute and subacute portal vein thrombosis. Tech Vasc Interv Radiol 2016;19:42-51.

45 Han G, Qi X, He C et al. Transjugular intrahepatic portosystemic shunt for portal vein thrombosis with symptomatic portal hypertension in liver cirrhosis. J Hepatol 2011;54:78-88.
46 Luca A, Miraglia R, Caruso S et al. Short- and long-term effects of the transjugular intrahepatic portosystemic shunt on portal vein thrombosis in patients with cirrhosis. Gut 2011;60:846-52.

47 Senzolo M, Tibbals J, Cholongitas E et al. Transjugular intrahepatic portosystemic shunt for portal vein thrombosis with and without cavernous transformation. Aliment Pharmacol Ther 2006;23:76775 .

48 Tripodi A, Mannucci PM. The coagulopathy of chronic liver disease. N Engl J Med 2011;365:147-56.

49 Northup PG, Caldwell SH. Coagulation in liver disease: a guide for the clinician. Clin Gastroenterol Hepatol 2013;11:1064-74.

50 Spahr L, Boehlen F, de Moerloose P, Hadengue A. Anticoagulants in portal vein thrombosis: don't be so shy! Blood 2009;113:5031-2.

51 Martinelli I, Franchini M, Mannucci PM. How I treat rare venous thromboses. Blood 2008;112:4818-23.

52 Confer BD, Hanouneh I, Gomes M, Alraies MC. Q: is anticoagulation appropriate for all patients with portal vein thrombosis? Cleve Clin J Med 2013;80:611-3.

Address for correspondence: Dr Jecko Thachil, Department of Haematology, Manchester Royal Infirmary, Oxford Road, Manchester M13 9WL, UK

Email: jecko.thachil@cmft.nhs.uk 